Cultural complexity and demography: the case of folktales

Alberto Acerbi ${ }^{* a}$, Jeremy Kendal ${ }^{\text {b }}$, Jamshid J Tehrani ${ }^{\text {b }}$

a: School of Innovation Science, Eindhoven University of Technology, 5600 MB, Eindhoven,

The Netherlands

b: Centre for the Coevolution of Biology and Culture, Department of Anthropology,

University of Durham, South Road, Durham DH1 3LE, UK

*: Corresponding author: alberto.acerbi@gmail.com

Word count: 6,625 


\title{
Cultural complexity and demography: the case of folktales
}

\begin{abstract}
We investigate the relationship between cultural complexity and population size in a nontechnological cultural domain for which we have suitable quantitative records: folktales. We define three levels of complexity for folk narratives: the number of tale types, the number of narrative motifs, and, finally, the number of traits in variants of the same type, for two well known tales for which we have data from previous studies. We found a positive relationship between number of tale types and population size, a negative relationship for the number of narrative motifs, and no relationship for the number of traits. The absence of a consistent relationship between population size and complexity in folktales provides a novel perspective on the current debates in cultural evolution. We propose that the link between cultural complexity and demography could be domain dependent: in some domains (e.g. technology) this link is important, whereas in others, such as folktales, complex traditions can be easily maintained in small populations as well as large ones, as they may appeal to universal cognitive biases.
\end{abstract}

\section{Keywords}

Cultural evolution; cultural complexity; demography; folktales; cultural attraction; content biases. 


\section{Introduction}

Recent work in cultural evolutionary theory has explored the relationship between demography (in particular, population size) and cultural complexity (French, 2015; Henrich, 2004; Shennan, 2015). Formal models, both involving selectively neutral (Premo \& Kuhn, 2010; Shennan, 2001) and non-neutral traits (Henrich, 2004; Kobayashi \& Aoki, 2012; Powell, Shennan, \& Thomas, 2009; Shennan, 2001), predict that population size affects a population's ability to invent and maintain complex culture (but see, for a criticism to this approach: Querbes, Vaesen, \& Houkes, 2014; Vaesen, 2012; Andersson \& Read, 2016; Vaesen, Collard, Cosgrove, \& Roebroeks, 2016, 2016a).

These models are based on two widely shared intuitions: that small societies, due to having fewer inventors, have lower rates of invention; and that, in the rare event of invention, innovations are more likely to be lost in smaller populations, simply as a result of random loss or incomplete transmission (Richerson, Boyd, \& Bettinger, 2009). For example, an influential model developed by anthropologist Joe Henrich (Henrich, 2004) proposes that, in any given population, individuals will attempt to copy the most accomplished demonstrator of a particular skill, but, since social learning is error prone, on average, learners would not be expected to attain the level of skill of the demonstrator, with only a small chance of equalling or surpassing him/her. Consequently, in a small population, it is rare that complex traits (for which errors are more likely) will be copied correctly, resulting in a loss of cultural complexity. "Population size" in this model is intended as the number of individuals that are potentially able to interact, and is referred to as "effective population size" to distinguish it from "census population size", i.e. census data on the estimated total number of individuals belonging to a particular ethno-linguistic group (Henrich et al., 2016, see also Lycett \& Norton, 2010 for a similar definition). It has been pointed out that for cultural traits, the true 
"effective population size" may vary from a census count due to the possibility of cultural exchanges across ethno-linguistic boundaries (Henrich et al., 2016).

The existence of a positive effect of population size on cultural complexity is supported by a growing body of results from laboratory experiments in which larger groups of participants are able to create, and support, more complex culturally-transmitted behaviours than smaller groups (Derex, Beugin, Godelle, \& Raymond, 2013; Derex \& Boyd, 2015; Kempe \& Mesoudi, 2014; Muthukrishna, Shulman, Vasilescu, \& Henrich, 2014). In parallel, a number of empirical studies have explored the existence of a correlation between cultural complexity and population size. These studies generally focused on subsistencerelated technologies (see e.g. Buchanan, O’Brien, \& Collard, 2015; Collard, Buchanan, \& O’Brien, 2013; Collard, Buchanan, O’Brien, \& Scholnick, 2013; Collard, Kemery, \& Banks, 2005; Collard, Ruttle, Buchanan, \& O’Brien, 2013; Kline \& Boyd, 2010; Read, 2008). The majority of cultural evolutionists consider the results of these analyses to provide robust support for a positive correlation between cultural complexity and population size (Henrich et al., 2016), although some researchers remain skeptical (Andersson \& Read, 2016; Vaesen et al., 2016, 2016a).

While the relationship between demography and cultural complexity has been a key debate in the field of cultural evolution, the evidence produced by the empirical studies above is restricted to the domain of technology. Some studies have explored how linguistic complexity is influenced by demographic variables, but the results remain contentious (Hay \& Bauer, 2007; Lupyan \& Dale, 2010; Moran, McCloy, \& Wright, 2012; Roberts \& Winters, 2012). To our knowledge, no studies have explored, from a cultural evolutionary perspective, how population size might influence cultural complexity in other non-technological domains, where the same intuitions about social learning might be expected to apply. Here, we investigate one such domain, for which we have suitable quantitative information: folktales. 
A folktale is defined as a prose narrative that cannot be attributed to any individual author, but, rather, constitutes a shared cultural tradition that has been passed on from person to person, and from generation to generation, usually by means of oral transmission (Thompson, 1951). While these stories will be familiar to many or most members of a population, literary and ethnographic research suggests that their long-term transmission depends on a small percentage of "active bearers" - expert storytellers who are directly analogous to "skilled demonstrators" in models of technological transmission (Henrich, 2004) - without whom these traditions would rapidly degenerate (e.g. Hansen, 2002; Sydow, 1948). Based on the demographic models discussed above, we might therefore expect the complexity of folk narrative traditions to covary with the number of active bearers available in a population. This is because when an individual invents a new tale or elaborates on an existing one (e.g. by introducing new characters an events) their innovations are more likely to catch on when there are other individuals who are sufficiently talented to memorise and reproduce them, in a manner directly analogous to the accumulation of technological complexity.

In what follows, using both data from the Aarne Thompson Uther (ATU) Index and from previous phylogenetic analysis of two tales, we analyse three levels of cross-cultural complexity in folk narrative, based on: (i) the number of "tale types", (ii) the number of narrative "motifs", and (iii) the number of traits in different variants of the same "type". 


\section{Material and methods}

We define three levels of complexity in folk narrative.

\subsection{The number of "tale types"}

A tale type represents an independent (i.e. self-contained), stable, storyline constituted by a specific combination of core narrative elements known as "motifs" (e.g. a human marries an animal, or a hero is set impossible tasks). Data on tale type distributions were drawn from the Aarne Thompson Uther (ATU) Index, a catalogue of over 2,000 "tale types" recorded in over 200 societies worldwide. We limited our analysis to the two most widespread genres of tale types in the ATU Index, "Animal Tales" (featuring non-human protagonists, as typified by Aesop's fables) and "Tales of Magic" (concerning beings or objects with supernatural powers, such as fairies, witches or magic rings). We considered only European and western Asian populations, to avoid (or limit) the effects of sampling biases, as the folktale traditions of these populations are better represented in the ATU sample than those of less well-studied groups. Figure 1 shows, as an example of our data, the approximate geographical location, and the quantity, of "Animal Tales" used in our analysis.

We extracted contemporary population size data in Wikipedia, searching for the population/ethnic group page, i.e. not the language or the actual country (see e.g. https://en.wikipedia.org/wiki/Italians). Where population data were reported as a range (e.g. https://en.wikipedia.org/wiki/English_people) we simply considered the average. Populations with ambiguous estimates were excluded. Population sizes were log-transformed, as the relationship between population size and cultural complexity is predicted to be concave by the demographic hypothesis (e.g. Collard, Buchanan, \& O’Brien, 2013). It is worth noting, however, that log-transforming the data does not change the qualitative result of the analysis. In addition to contemporary population sizes, we also calculated log-transformed population 
estimates at the end of the nineteenth century using information available from Wikipedia (https://en.wikipedia.org/wiki/List_of_countries_by_population_in_1900). These estimates provide a useful point of comparison for analysing the relationship between folktale complexity and demography, since many of the sources used in the ATU Index date back to the late nineteenth or early twentieth centuries (notice also that end of the nineteenth century population size estimates strongly correlate with contemporary estimates). Our final sample comprised 380 different Animal Tales types in 73 contemporary populations and 24 nineteenth century populations, and 276 different Tales of Magic types in 74 contemporary populations and 26 nineteenth century populations. Full data are available as Supplementary material, table S1 (“Animal Tales") and table S2 ("Tales of Magic").

We fitted complexity and population size data with a Bayesian multilevel model with a Poisson likelihood (following McElreath, 2016). In order to control for phylogenetic and spatial autocorrelation, the model included parameters for "language family" (i.e. membership of linguistic families) and "region" using population codes from the crosscultural database D-Place (Kirby et al., 2016). For the most dominant language family in the sample, Indo-European, the phylogenetic control was coded at the level of linguistic subfamilies. The same procedure was applied in all the following analyses. The $\mathrm{R}$ code to perform the analysis is provided in Supplementary material ("analysis.R") and it requires the R package "rethinking” (McElreath, 2016a).

\subsection{The number of narrative "motifs"}

As explained above, motifs are the basic building blocks of tale types. We used the motif typology developed by Thompson (1955) on which the ATU Index is based, focusing again on European and western Asian populations. Although the distribution of motifs is closely tied to the cross-cultural patterning of tale types, these two measures of complexity are not 
synonymous. For example, a population may have a large number of stories created recombining a small number of motifs, while another may have a relatively small number of tale types but each using a large number of unique motifs.

For this analysis, we focused on "Tales of Magic", since these tales typically contain more motifs than other genres (for instance, the majority of Animal Tales comprise one or two motifs, with a maximum of five, whereas the maximum number of motifs in Magic Tales is 20 , with a mean of 7.2 per tale). Once again, we only considered European and western Asian populations for the reasons given above, giving a total of 74 populations and 5,664 tales (notice in this case the same tale type can be present in different societies), and 2,471 tales and 26 nineteenth century populations. For each tale, we calculated how many ATU motifs were present (data extracted from Ofek, Dará nyi, \& Rokach 2013, full data available as Supplementary material, table S3). For this analysis, together with "language family" and "region", "society" was included as a parameter of the multilevel model.

\subsection{The number of "traits" in variants of the same type}

Variants of a tale type largely share the same motifs, but vary in their specific details or "traits". For example, the Grimm brothers' "Rotkappchen" and Charles Perrault's "Petit Chaperon Rouge" are both variants of the same tale type, "Little Red Riding Hood", but contain distinctive features, such as the rescue of the little girl from the wolf's stomach in the Grimm tale (absent in Perrault's version). The complexity of variants recorded for any given tale type in different populations can be measured by the average number of traits they exhibit. We considered variants of two different tales, using data from two previous studies.

\subsubsection{Little Red Riding Hood}


We obtained data on 72 traits exhibited by 58 variants of "Little Red Riding Hood" from Tehrani (2013). We considered for our analysis only traits that could be recoded in terms of presence/absence, e.g. we excluded traits such as the number 1 in Tehrani table ("Species of the victim: [0] animal [1] human") as they do not make intuitively a difference in the complexity of the variant. Multiple possibilities were also recoded as present/absent (e.g. trait 11 "The setting: [0] absent [1] woods [2] mountains [3] cave" was recoded as "The setting: [0] absent [1-2-3] present”). This yielded a total of 59 different traits. For this analysis, as well as the two that follow, population size data were based on the closest estimates available for the time at which a given variant was recorded. One of the variant was assigned to "Unknown" population in Tehrani (2013) and for another one we did not find unambiguous population size data, so our analysis considered a total of 56 variants. As in the previous analyses, we also considered nineteenth century population size data as benchmark. For this latter analysis, our sample was composed by 27 variants. The complexity of each variant was calculated as the number of present traits. Full data are available as Supplementary material, table S4.

\subsubsection{The tale of the kind and the unkind girls}

We obtained data on different variants of "The tale of the kind and the unkind girls" from Ross, Greenhill, \& Atkinson (2013). Ross and colleagues analysed a vast dataset of 700 variants (coming from 31 different populations), and 424 narrative traits, sourced from Roberts (1958). Traits were already coded as present/absent, so we did not process the data any further. The additional analysis with nineteenth century population size data comprised 491 variants. The complexity of variants was calculated as before. However, for "The tale of the kind and the unkind girls", unlike the dataset of "Little Red Riding Hood", we have several variants for each population. For this reason, we added "society" as a parameter of the 
model (analogously to the analysis of the number of narrative motifs, see section 2.2 above). Full data are available as Supplementary material, table S5.

\section{Results}

Table 1 summarises the main results of our models. The output we are interested in is $\beta$, which represents the "slope" of the regression complexity/population size. Following McElreath (2016), we report the 5.5\% and $94.5 \%$ quantiles of the posterior probability, and we consider that, when these (arbitrary-sized) $89 \%$ intervals do not overlap zero, this provides strong evidence of a relationship between complexity and population size. Three out of five measures were affected by population size, but, in one case, population size was negatively associated to the complexity of folktales. More specifically, the two measures of complexity defined by the number of tale types were positively related to population size (see also Figure 2), and the measure defined by the number of narrative motifs was negatively associated with population size (see also Figure 3). For the other two measures, pertaining to the number of traits in different variants of the two tales "Little Red Riding Hood" and "The tale of the kind and the unkind girls", a relationship between population size and complexity is incompatible with the data, as the $89 \%$ intervals overlap zero (see also Figure 4).

The same analysis, applied to population size estimated for the nineteenth century, gave qualitatively similar results (see Table 2, and Supplementary material “Figures_popSize1900.pdf”). All posterior probability intervals were, for these estimates, noticeably broader, as fewer data points were present.

\section{Discussion}

The results of our analyses provide conflicting evidence about the relationship between demography and the complexity of oral traditions. On the one hand, we found strong support 
for a positive relationship between population size and the overall number of folktales recorded in different societies. On the other, it appears that the tales told in larger populations have a lower average number of motifs than those in smaller groups, while a comparison of variants of two international tale types found no consistent relationship between population size and the number of characters, events and other plot traits.

To explain this apparent conflict, it is important to consider that different measures can capture different levels of cultural complexity: while the number of tale types maintained within a population is potentially a measure of cultural accumulation (i.e. the invention and retention of many different kinds of stories within a single, unified tradition), it could also be a reflection of cultural variation within that population (multiple overlapping traditions from different localities, each of which may consist of a relatively small numbers of stories). Since larger populations generally occupy larger geographic areas, it is possible that the larger number of tales recorded for these groups reflects regional diversity in folktales, rather than the richness of a shared corpus. Another potentially relevant factor is that the folktale traditions of larger populations might have been more extensively studied than those of smaller groups, leading to sampling biases in the number of tales included for each population in the ATU Index.

The second and third sets of analyses focused on storyline architectures, rather than simply the number of different stories. For these analyses neither the number of motifs nor plot traits in tale type variants should be sensitive to the amount of regional diversity within populations or sampling bias in the ATU. From this perspective, the negative relationship we found between the number of narrative motifs and population size could reflect another level of cultural complexity. In larger populations there could be a stronger pressure for folktales to be learnable by a larger number of individuals, favouring compressibility versus expressivity, 
and ultimately simplicity, as already proposed for languages (see e.g. Lupyan \& Dale, 2010; Kirby, Tamariz, Cornish, \& Smith, 2015).

The results for the measures of complexity related to the number of traits in the two tales we analysed in details, for which we did not find an indication of a relationship with population size, leave open the question of whether this might be due to data insensitivity. In other terms: is the absence of relationship simply due to the fact that we did not consider enough data points in our analysis? We cannot solve this question with the current analysis, but we can point to the width of the posterior probability for the parameter $\beta$ (see Table 1). Broad distributions may indicate that more data are needed, while narrow distributions (around zero) suggest more strongly an absence of relationship.

Another possible limitation in our study is that, following a number of other similar studies (e.g. Buchanan, O’Brien, \& Collard, 2015; Collard, Buchanan, O’Brien, \& Scholnick, 2013), we measured population size using census data on the estimated total number of individuals belonging to a particular ethno-linguistic group, and this may not correspond exactly with the "effective population size" (Henrich et al., 2016; Lycett and Norton, 2010). Indeed, Ross \& Atkinson (2016) suggest that the distributions of shared folktales among ethnically diverse Arctic hunter-gatherers provide evidence for "high bandwidth" social learning extending over thousands of kilometres. However, while there can be no doubt about the potential for folktales to circulate among populations over very large distances, there is evidence to suggest that language barriers and cultural differences between groups act to contain the transmission of folktale traditions among the less multilingual, more sedentary, higher-density populations of Europe and western Asia. For example, da Silva \& Tehrani (2016) spatial and phylogenetic analyses of ATU tale type distributions in Indo-European groups suggested that geographic proximity is often negatively correlated with the sharing of tales, suggesting that these populations may consciously reject stories associated with their 
neighbours. Meanwhile, Ross et al.'s (2013) investigation into patterns of variation in "The tale of the kind and the unkind girls" found that variants separated by a linguistic/cultural barrier were 10 times more different from one another than variants separated by an equivalent geographical distance within the same culture. Taken together, these studies suggest that census data on ethno-linguistic groups provide a reasonable proxy of effective cultural population size - especially in the absence of any alternative measure that could be easily operationalized.

Overall, we suggest that the absence of a consistent relationship between population size and complexity in folktales may provide a novel perspective on the current debate in cultural evolution (Henrich et al., 2016; Vaesen et al., 2016). Models like the Henrich's, mentioned in the Introduction (Henrich, 2004; Powell et al., 2009), are based on reasonable assumptions that are standard in cultural evolutionary theory: that copying is a process of selection among variants, and that it is subject to random errors, the majority of which will deteriorate the trait one attempts to copy. However, both these assumptions have been challenged by an alternative approach to cultural evolution, known as cultural attraction (Claidière, Scott-Phillips, \& Sperber, 2014; Claidière \& Sperber, 2007; Morin, 2015; Sperber, 1996). Proponents of cultural attraction believe that cultural transmission is not a process of selection and copying, but primarily a process of reconstruction, and, importantly, that modifications are in general not random, but oriented towards particular "attractors" (hence the name of the approach).

Oral literature has long been considered to be a domain in which transmission is influenced by cultural attractors, as exemplified by Sperber's "law" of oral tradition: "In an oral tradition, all cultural representations are easily remembered ones; hard-to-remember representations are forgotten, or transformed into more easily remembered ones before reaching a cultural level of distribution" (Sperber, 1996: 74). Laboratory recreations of oral 
traditions using transmission chain experiments (Bartlett, 1932; Mesoudi \& Whiten, 2008) demonstrate that the stability and distortion of narratives are influenced by a number of psychological biases, defined in cultural evolution as content biases. These include: i) a bias for survival-relevant information, such as predators, the location of food, access to resources, reproductive strategies (e.g. Stubbersfield, Tehrani, \& Flynn, 2015); ii) a bias for social information, e.g. about peoples' relations and interactions with one another (e.g. Mesoudi, Whiten, \& Dunbar, 2006; Stubbersfield, Tehrani, \& Flynn, 2015); iii) a bias for emotionally arousing content, such as disgust, or amusement (Eriksson \& Coultas, 2014; Stubbersfield, Tehrani, \& Flynn, 2017); iv) a bias for minimally-counterintuitive (MCI) information that violates some of our implicit ontological assumptions about the world in ways that makes it more salient than information that can either be taken-for-granted or is incomprehensible (e.g. Barrett \& Nyhof, 2001); v) a stereotype-consistency bias, in which information is transformed and recalled in ways that make it more consistent with pre-existing expectations and prejudices (e.g. Bangerter, 2000).

These findings challenge the widely-held, but often implicit, assumption that oral transmission is necessarily messy and error-prone. Even some folktale scholars (e.g. Bottigheimer 1993, 2009) have suggested that the long-term survival of traditional narratives probably depends on the support of literary texts, without which they would be liable to degenerate. While there is no doubt that oral variants of some tales have been significantly influenced by textual versions, it seems improbable that the cross-cultural distributions of tale types and motifs can be explained by literary dissemination. First, there is no literary tradition for the majority of international tale types that predates the oral versions collected by folklorists in the nineteenth and twentieth centuries (Vaz da Silva 2010). Second, both qualitative content analyses (Ben Amos et al. 2010) and quantitative phylogenetic studies (da Silva \& Tehrani 2016, Tehrani 2013, Tehrani et al. 2016) suggest that these tales probably 
existed in oral tradition long before the emergence of literary fairy tales in the seventeenth and eighteenth centuries. Cultural attraction theory provides an alternative explanation for how folktales survive the supposed vagaries of oral transmission that does not require the existence of a supporting literary record, but focuses instead on the psychological and ecological factors that stabilise narratives into forms that are more appealing and easier to remember.

At the same time, cultural attraction might explain the lack of a consistent correlation between folktale complexity and population size found in our study. If modifications are not random, but directed, and hence there are no particular reasons to assume that a cultural trait will deteriorate, the correlation between demography and cultural complexity appears intuitively less strong. While bigger populations can explore the space of possible cultural traits more thoroughly_ or faster-, once an attractor is found then a small population will also be able to preserve traits in its "basin of attraction", because individuals, even assuming imperfect copying, will tend to reproduce the features associated with the attractor.

Note we are not proposing that the effects of demography on cultural complexity and preservation are not important in general, but that they instead may be domain-dependent. One can conceive different cultural domains on a continuum (Acerbi \& Mesoudi, 2015) in which, at one extreme, cultural maintenance heavily depends on high-fidelity transmission, errors are random and, on average, deleterious. This is likely to be the case, for example, of technology: on this end of the continuum, cultural reproduction is more subject to dynamics analogous to the ones described in Henrich (2004)'s model, and demography might be an important determinant of cultural complexity. On the other end of the continuum, instead, we find domains such as folktales, where modifications are non-random, as some features tend to be reconstructed with higher probability than others. Here one would not expect, for the reasons explained above, a correlation between population size and cultural complexity. An 
analogous way to think about this distinction is in terms of different search landscapes: some domains, like technology, are characterised by narrow-peaked search spaces, where optimal solutions need to be found with the help of high-fidelity social learning. Others, like folktales, are characterised by smooth search spaces, and individuals can relatively easily reproduce the features of cultural traits they are attempting to copy (Acerbi, Tennie, \& Mesoudi, 2016).

Additional cultural domains that are likely to possess similar characteristics are, for example, kinship systems, which have been shown to be constrained by universal principles that make them efficient for communication, balancing simplicity and informativeness (Kemp \& Regier, 2012), or some aspects of religious systems, like psychological features of supernatural agents, also considered to be reconstructed, at each instance of transmission, according to universal cognitive constraints (Barrett, 2000; Boyer, 2001).

Future studies may investigate whether these domains exhibit - or, as we predict, fail to exhibit - a correlation between population size and complexity. More generally, the fact that the importance of reconstruction (plus non-random modifications) and high-fidelity copying (plus random, detrimental, errors) differ in different cultural domains may help to explain possible gaps of the empirical support for the existence of an overall correlation between cultural complexity and population size.

\section{Acknowledgements}

We thank Sá ndor Dará nyi and Nir Ofek for providing the data on motif distributions, and Robert Ross for providing the data used in the analysis of "The tale of the kind and unkind girls". We also thank Eugenio Bortolini, Thom Scott-Phillips, Krist Vaesen, and two anonymous reviewers for comments on previous versions of the manuscript. 


\section{Data availability}

The data associated with this research are available as Supplementary material.

\section{Funding}

The work of AA was supported by a grant from The Netherlands Organisation for Scientific Research (NWO VIDI-grant 016.144312).

\section{References}

Acerbi, A., Tennie, C., \& Mesoudi, A. (2016). Social learning solves the problem of narrowpeaked search landscapes: experimental evidence in humans. Royal Society Open Science, 3(1), 160215. http://doi.org/10.1098/rsos.160215

Acerbi, A., \& Mesoudi, A. (2015). If we are all cultural Darwinians what's the fuss about? Clarifying recent disagreements in the field of cultural evolution. Biology \& Philosophy, 30(4), 481-503. http://doi.org/10.1007/s10539-015-9490-2

Andersson, C., \& Read, D. (2016). The Evolution of Cultural Complexity: Not by the Treadmill Alone. Current Anthropology. 57(3), 261-286. http://doi.org/10.1086/686317

Bangerter, A. (2000). Transformation between scientific and social representations of conception: The method of serial reproduction. British Journal of Social Psychology, 39(4), 521-535. http://doi.org/10.1348/014466600164615

Barrett, J. L. (2000). Exploring the natural foundations of religion. Trends in Cognitive Sciences, 4(1), 29-34. http://doi.org/10.1016/S1364-6613(99)01419-9

Barrett, J., \& Nyhof, M. (2001). Spreading Non-natural Concepts: The Role of Intuitive Conceptual Structures in Memory and Transmission of Cultural Materials. Journal of Cognition and Culture, 1(1), 69-100. http://doi.org/10.1163/156853701300063589

Bartlett, F. C. (1932). Remembering $\square$ : a study in experimental and social psychology. 
Cambridge: Cambridge University Press.

Ben-Amos, D., Ziolkowski, J. M. , Silva, F. V. D. , \& Bottigheimer, R. (2010) Special issue: the European fairy-tale tradition between orality and literacy. The Journal of American Folklore, 123.

Boyer, P. (2001). Cognitive templates for religious concepts: cross-cultural evidence for recall of counter-intuitive representations. Cognitive Science, 25(4), 535-564. http://doi.org/10.1016/S0364-0213(01)00045-3

Bottigheimer, R. B. (1993). Luckless, Witless, and Filthy-Footed: A Sociocultural Study and Publishing History Analysis of 'The Lazy Boy'. The Journal of American Folklore, 106(421), 259-284.

Bottigheimer, R. B. (2009) Fairy Tales: A New History. New York: State University of New York Press.

Buchanan, B., O’Brien, M. J., \& Collard, M. (2015). Drivers of technological richness in prehistoric Texas: an archaeological test of the population size and environmental risk hypotheses. Archaeological and Anthropological Sciences, 1-10. http://doi.org/10.1007/s12520-015-0245-4

Claidière, N., Scott-Phillips, T. C., \& Sperber, D. (2014). How Darwinian is cultural evolution? Philosophical Transactions of the Royal Society B, 369(1642), 20130368. http://doi.org/10.1098/rstb.2013.0368

Claidière, N., \& Sperber, D. (2007). The role of attraction in cultural evolution. Journal of Cognition and Culture, 7(1), 89-111. http://doi.org/10.1163/156853707X171829

Collard, M., Buchanan, B., \& O’Brien, M. J. (2013). Population Size as an Explanation for Patterns in the Paleolithic Archaeological Record: More Caution Is Needed. Current Anthropology, 54(S8), S388-S396. http://doi.org/10.1086/673881

Collard, M., Buchanan, B., O’Brien, M. J., \& Scholnick, J. (2013). Risk, mobility or 
population size? Drivers of technological richness among contact-period western North American hunter-gatherers. Philosophical Transactions of the Royal Society of London. Series B, Biological Sciences, 368(1630), 20120412.

http://doi.org/10.1098/rstb.2012.0412

Collard, M., Kemery, M., \& Banks, S. (2005). Causes of Toolkit Variation Among HunterGatherers: A Test of Four Competing Hypotheses. Canadian Journal of Archaeology, 29(1), 1-19.

Collard, M., Ruttle, A., Buchanan, B., \& O’Brien, M. J. (2013). Population size and cultural evolution in nonindustrial food-producing societies. PloS One, 8(9), e72628. http://doi.org/10.1371/journal.pone.0072628

da Silva, S. G., \& Tehrani, J. J. (2016). Comparative phylogenetic analyses uncover the ancient roots of Indo-European folktales. Royal Society Open Science, 3(1), 150645. http://doi.org/10.1098/rsos.150645

Derex, M., Beugin, M.-P., Godelle, B., \& Raymond, M. (2013). Experimental evidence for the influence of group size on cultural complexity. Nature, 503(7476), 389-91. http://doi.org/10.1038/nature12774

Derex, M., \& Boyd, R. (2015). The foundations of the human cultural niche. Nature Communications, 6, 8398. http://doi.org/10.1038/ncomms9398

Eriksson, K., \& Coultas, J. C. (2014). Corpses, Maggots, Poodles and Rats: Emotional Selection Operating in Three Phases of Cultural Transmission of Urban Legends. Journal of Cognition and Culture, 14(1-2), 1-26. http://doi.org/10.1163/1568537312342107

French, J. C. (2015). Demography and the Palaeolithic Archaeological Record. Journal of Archaeological Method and Theory. http://doi.org/10.1007/s10816-014-9237-4

Grimm, W. (1884) Children's and household tales (3rd edn). London: George Bell. 
Hansen, W. (2002). Ariadne's Thread, A Guide to International Stories in Classical Literature. Ithaca: Cornell University Press.

Hay, J., \& Bauer, L. (2007). Phoneme inventory size and population size. Language, 83(2), 388-400. http://doi.org/10.1353/lan.2007.0071

Henrich, J. (2004). Demography and Cultural Evolution: How Adaptive Cultural Processes can Produce Maladaptive Losses: The Tasmanian Case. American Antiquity, 69(2), 197214.

Henrich, J., Boyd, R., Derex, Kline, M., Mesoudi, A., Muthukrishna, M., ... Thomas, M. G. (2016). Understanding Cumulative Cultural Evolution. Proceedings of the National Academy of Sciences of the United States of America, 113(44), E6724-E6725. http://doi: 10.1073/pnas.1610005113

Kemp, C., \& Regier, T. (2012). Kinship Categories Across Languages Reflect General Communicative Principles. Science, 336(6084), 1049-1054. http://doi.org/10.1126/science.1218811

Kempe, M., \& Mesoudi, A. (2014). An experimental demonstration of the effect of group size on cultural accumulation. Evolution and Human Behavior, 35(4), 285-290. http://doi.org/10.1016/j.evolhumbehav.2014.02.009

Kirby, K. R., Gray, R. D., Greenhill, S. J., Jordan, F. M., Gomes-Ng, S., Bibiko, H.-J., ... Gavin, M. C. (2016). D-PLACE: A Global Database of Cultural, Linguistic and Environmental Diversity. PloS One, 11(7), e0158391. http://doi.org/10.1371/journal.pone.0158391

Kirby, S., Tamariz, M., Cornish, H., \& Smith, K. (2015). Compression and communication in the cultural evolution of linguistic structure. Cognition, 141(1), 87-102. http://dx.doi.org/10.1016/j.cognition.2015.03.016

Kline, M. A., \& Boyd, R. (2010). Population size predicts technological complexity in 
Oceania. Proceedings. Biological Sciences / The Royal Society, 277(1693), 2559-64. http://doi.org/10.1098/rspb.2010.0452

Kobayashi, Y., \& Aoki, K. (2012). Innovativeness, population size and cumulative cultural evolution. Theoretical Population Biology, 82(1), 38-47. http://doi.org/10.1016/j.tpb.2012.04.001

Lycett, S. J., \& Norton, C. J. (2010). A demographic model for Palaeolithic technological evolution: the case of East Asia and the Movius Line. Quaternary International, 211 (1), 55-65. http://dx.doi.org/10.1016/j.quaint.2008.12.001

Lupyan, G., \& Dale, R. (2010). Language structure is partly determined by social structure. PloS One, 5(1), e8559. http://doi.org/10.1371/journal.pone.0008559

McElreath, R. (2016). Statistical rethinking: a Bayesian course with examples in R and Stan. Boca Raton: CRC Press

McElreath, R. (2016a). rethinking, R package version 1.56.

Mesoudi, A., \& Whiten, A. (2008). The multiple roles of cultural transmission experiments in understanding human cultural evolution. Philosophical Transactions of the Royal Society of London. Series B, Biological Sciences, 363(1509), 3489-501. http://doi.org/10.1098/rstb.2008.0129

Mesoudi, A., Whiten, A., \& Dunbar, R. (2006). A bias for social information in human cultural transmission. British Journal of Psychology, 97(3), 405-423. http://doi.org/10.1348/000712605X85871

Moran, S., McCloy, D., \& Wright, R. (2012). Revisiting population size vs. phoneme inventory size. Language, 88(4), 877-893. http://doi.org/10.1353/lan.2012.0087

Morin, O. (2015). How Traditions Live and Die. Oxford: Oxford University Press.

Muthukrishna, M., Shulman, B. W., Vasilescu, V., \& Henrich, J. (2014). Sociality influences cultural complexity. Proceedings. Biological Sciences / The Royal Society, 281(1774), 
20132511. http://doi.org/10.1098/rspb.2013.2511

Ofek, N., Darányi, S., \& Rokach, L. (2013) Linking Motif Sequences with Tale Types by Machine Learning. In Finlayson, M. A. Fisseni, B., Löwe, B., \& Meister, J. C.. editors, Proceedings of the 4th Workshop on Computational Models of Narrative (CMN'13), Hamburg, Germany, 2013. Schloss Dagstuhl-Leibniz-Zentrum fuer Informatik. doi:10.4230/OASIcs.CMN.2013.166.

Powell, A., Shennan, S., \& Thomas, M. G. (2009). Late Pleistocene demography and the appearance of modern human behavior. Science, 1298-1301. http://doi.org/10.1126/science.1170165

Premo, L. S., \& Kuhn, S. L. (2010). Modeling effects of local extinctions on culture change and diversity in the paleolithic. PloS One, 5(12), e15582. http://doi.org/10.1371/journal.pone.0015582

Querbes, A., Vaesen, K., \& Houkes, W. (2014). Complexity and demographic explanations of cumulative culture. PloS One, 9(7), e102543. http://doi.org/10.1371/journal.pone.0102543

Read, D. (2008). An Interaction Model for Resource Implement Complexity Based on Risk and Number of Annual Moves. American Antiquity, 73(4), 599-625.

Richerson, P. J., Boyd, R., \& Bettinger, R. L. (2009). Cultural Innovations and Demographic Change. Human Biology, 81(2-3), 211-235. http://doi.org/10.3378/027.081.0306

Roberts, W. E. (1958). The tale of the kind and the unkind girls: Aa-Th 480 and related tales. Berlin: De Gruyter.

Roberts, S., \& Winters, J. (2012). Social Structure and Language Structure: the New Nomothetic Approach. Psychology of Language and Communication, 16(2), 89-112. http://doi.org/10.2478/v10057-012-0008-6

Ross, R. M., \& Atkinson, Q. D. (2016). Folktale transmission in the Arctic provides evidence 
for high bandwidth social learning among hunter-gatherer groups. Evolution and Human Behavior, 37(1), 47-53. http://doi.org/10.1016/j.evolhumbehav.2015.08.001

Ross, R. M., Greenhill, S. J., \& Atkinson, Q. D. (2013). Population structure and cultural geography of a folktale in Europe. Proceedings. Biological Sciences / The Royal Society, 280(1756), 20123065. http://doi.org/10.1098/rspb.2012.3065

Shennan, S. (2001). Demography and Cultural Innovation: a Model and its Implications for the Emergence of Modern Human Culture. Cambridge Archaeological Journal, 11(1). http://doi.org/10.1017/S0959774301000014

Shennan, S. (2015). Demography and Cultural Evolution. Emerging Trends in the Social and Behavioral Sciences. http://doi.org/10.2307/4128416

Sperber, D. (1996). Explaining Culture: A Naturalistic Approach. Oxford: Wiley.

Stubbersfield, J. M., Tehrani, J., \& Flynn, E. G. (2017). Chicken Tumours and a Fishy Revenge: Evidence for Emotional Content Bias in the Cultural Transmission of Urban Legends. Journal of Cognition and Culture. http://doi.org/10.13140/RG.2.1.5181.0967

Stubbersfield, J. M., Tehrani, J. J., \& Flynn, E. G. (2015). Serial killers, spiders and cybersex: Social and survival information bias in the transmission of urban legends. British Journal of Psychology, 106(2), 288-307. http://doi.org/10.1111/bjop.12073

Sydow, C. (1948). Selected papers on folklore. Copenhagen: Rosenkilde and Bagger.

Tehrani, J. J. (2013). The phylogeny of Little Red Riding Hood. PloS ONE, 8(11), e78871. http://doi.org/10.1371/journal.pone.0078871

Tehrani, J. J., Nguyen, Q. \& Roos, T. (2016). Oral Fairy Tale or Literary Fake? Investigating the Origins of Little Red Riding Hood Using Phylogenetic Network Analysis. Digital Scholarship in the Humanities, 31(3), 611-636. http://dx.doi.org/10.1093/llc/fqv016 Thompson, S. (1951). The folktale. New York: Dryden. Thompson, S. (1955). Motif-index of Folk-literature: A Classification of Narrative Elements 
in Folktales, Ballads, Myths, Fables, Mediaeval Romances, Exempla, Fabliaux, Jestbooks and Local Legends. Bloomington: Indiana University Press.

Vaesen, K. (2012). Cumulative cultural evolution and demography. PloS One, 7(7), e40989. http://doi.org/10.1371/journal.pone.0040989

Vaesen, K., Collard, M., Cosgrove, R., \& Roebroeks, W. (2016). Population size does not explain past changes in cultural complexity. Proceedings of the National Academy of Sciences of the United States of America, 113(16), E2241-E2247.

http://doi.org/10.1073/pnas.1520288113

Vaesen, K., Collard, M., Cosgrove, R., \& Roebroeks, W. (2016a). Reply to Henrich et al.: The Tasmanian effect and other red herrings. Proceedings of the National Academy of Sciences of the United States of America, 113(44), E6726-E6727. http://10.1073/pnas.1613074113

Vaz da Silva., F. (2010). The invention of fairy tales. Journal of American Folklore. 123 (490), 398-425

Zipes J. (2013). The golden age of folk and fairy tales: from the brothers Grimm to Andrew Lang. Indianapolis: Hackett Publishing. 
Table 1

Measure of complexity

$\beta$

$5.5 \%$

94.5\% Relationship

Number of tale types:

Animal tales

Tales of magic

Number of motifs:

Tales of magic

Number of traits:

Little Red Riding Hood

The tale of the kind and the unkind girls
0.39

0.35

$0.41+$

0.21

0.19

$0.24+$

$-0.05$

$-0.07$

$-0.03-$

$\begin{array}{lll}0.0 & -0.07 \smile 0.06\end{array}$

0.04

$-0.05$

0.12

Table 1: Summary of results. Bold fonts indicate that the model provide strong support for the existence of a relationship between the measure of folktales complexity and population size. 
Table 2

Measure of complexity

$\boldsymbol{\beta}$

$5.5 \%$

94.5\% Relationship

Number of tale types:

Animal tales

$0.72 \quad 0.63$

0.82

$+$

Tales of magic

0.46

0.41

0.53

$+$

Number of motifs:

Tales of magic

$-0.08 \quad-0.11$

$-0.05$

Number of traits:

Little Red Riding Hood

$0.18 \quad-0.01$

0.36

The tale of the kind and the unkind girls

0.17

$-0.02$

0.33

Table 2: Summary of results for models using nineteenth century estimates of population size. Bold fonts indicate that the model provide strong support for the existence of a relationship between the measure of folktales complexity and population size. 


\section{Figure captions}

\section{Figure 1. ATU “Animal Tales".}

Visualisation of the approximate geographical location, and the quantity, of the tale types used in the analysis for the ATU genre "Animal Tales".

Figure 2. Number of tale types versus population size.

Complexity calculated as the number of tale types versus logarithm of population size, for which our model supports the existence of a positive relationship. A regression line and a $67 \%$ plausible interval, i.e. the region were the model expect to find the $67 \%$ of complexity, for each population size, are added to the plots. Left: ATU “Animal Tales". Right: ATU "Tales of Magic".

\section{Figure 3. Number of motifs versus population size.}

Complexity calculated as the average number of motifs for "Tales of Magic" versus logarithm of population size, for which our model supports the existence of a negative relationship. A regression line and a $67 \%$ plausible interval, i.e. the region were the model expect to find the $67 \%$ of complexity, for each population size, are added to the plot.

\section{Figure 4. Number of traits versus population size.}

Complexity calculated as the number of traits in different variants of the same tale type versus logarithm of population size, for which our model does not support the existence of a relationship. A regression line and a $67 \%$ plausible interval, i.e. the region were the model expect to find the $67 \%$ of complexity, for each population size, are added to the plots. Left: "Little Red Riding Hood". Right: "The tale of the kind and unkind girls". 


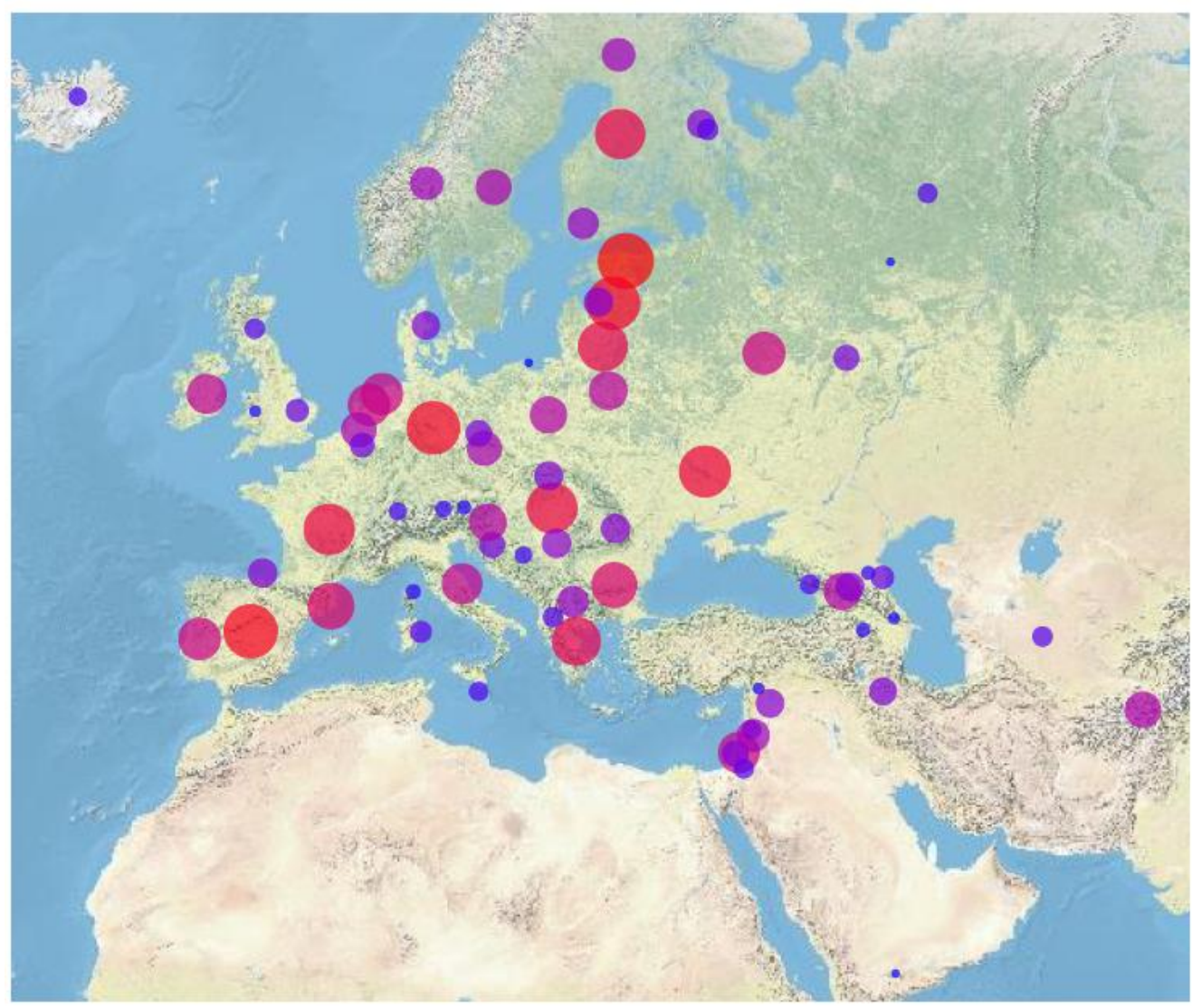

Number of tales

- 10

60

( 110

160

Figure 1

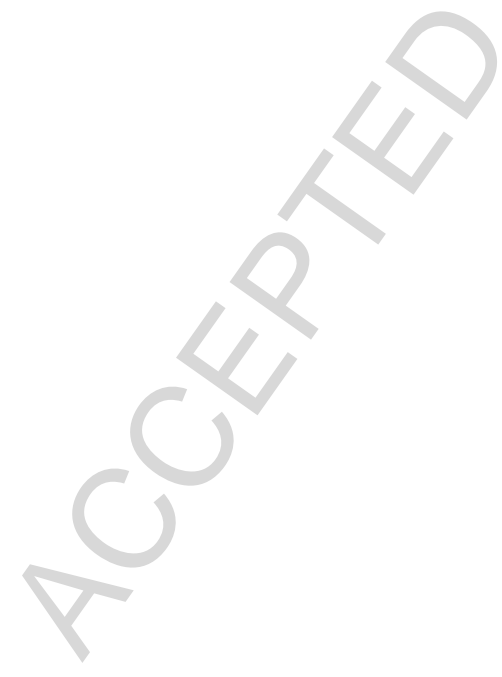



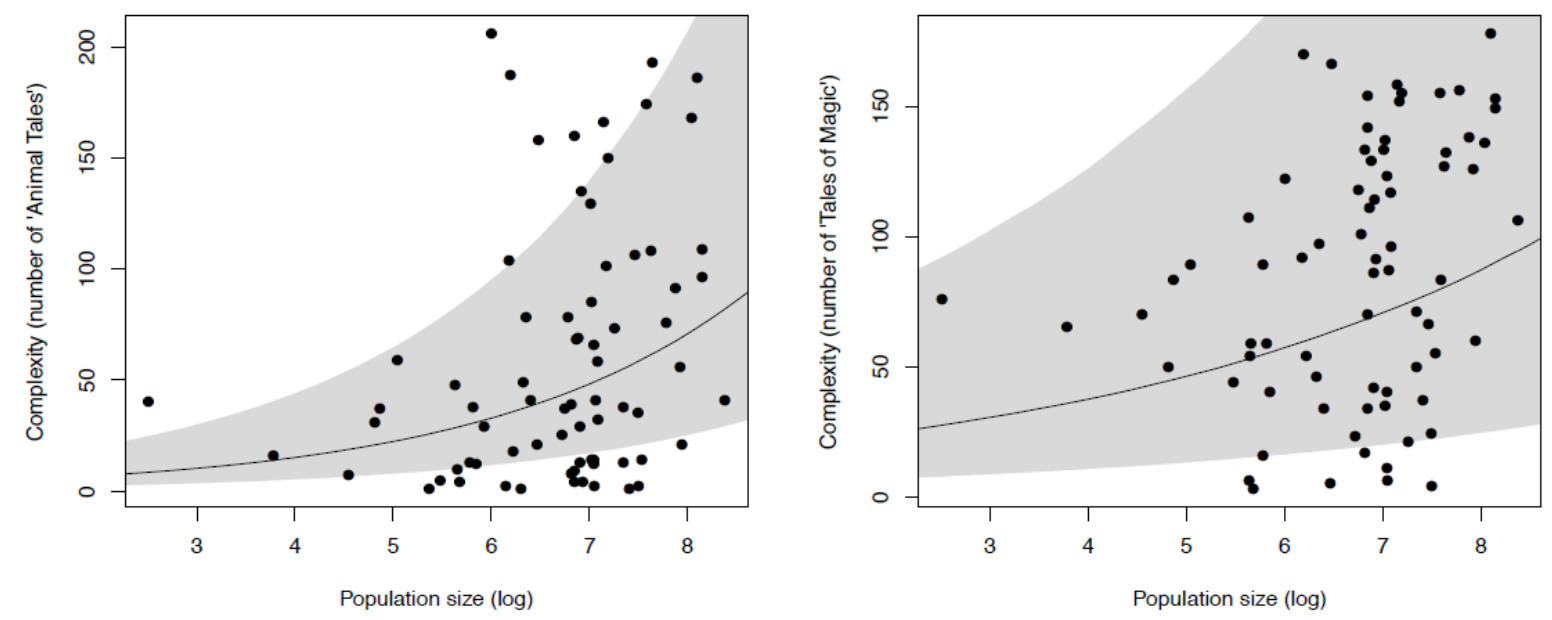

Figure 2 


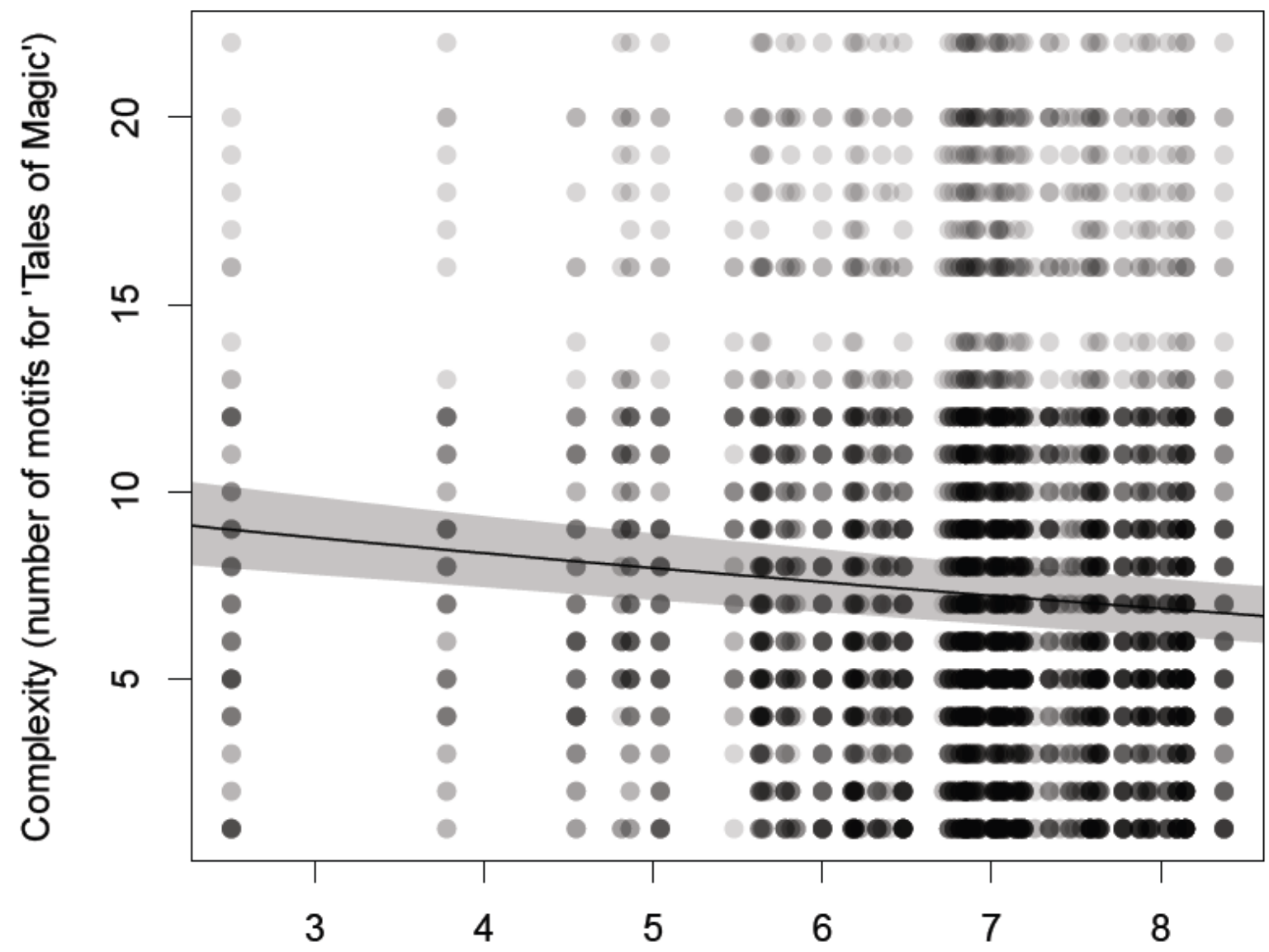

Population size (log)

Figure 3 

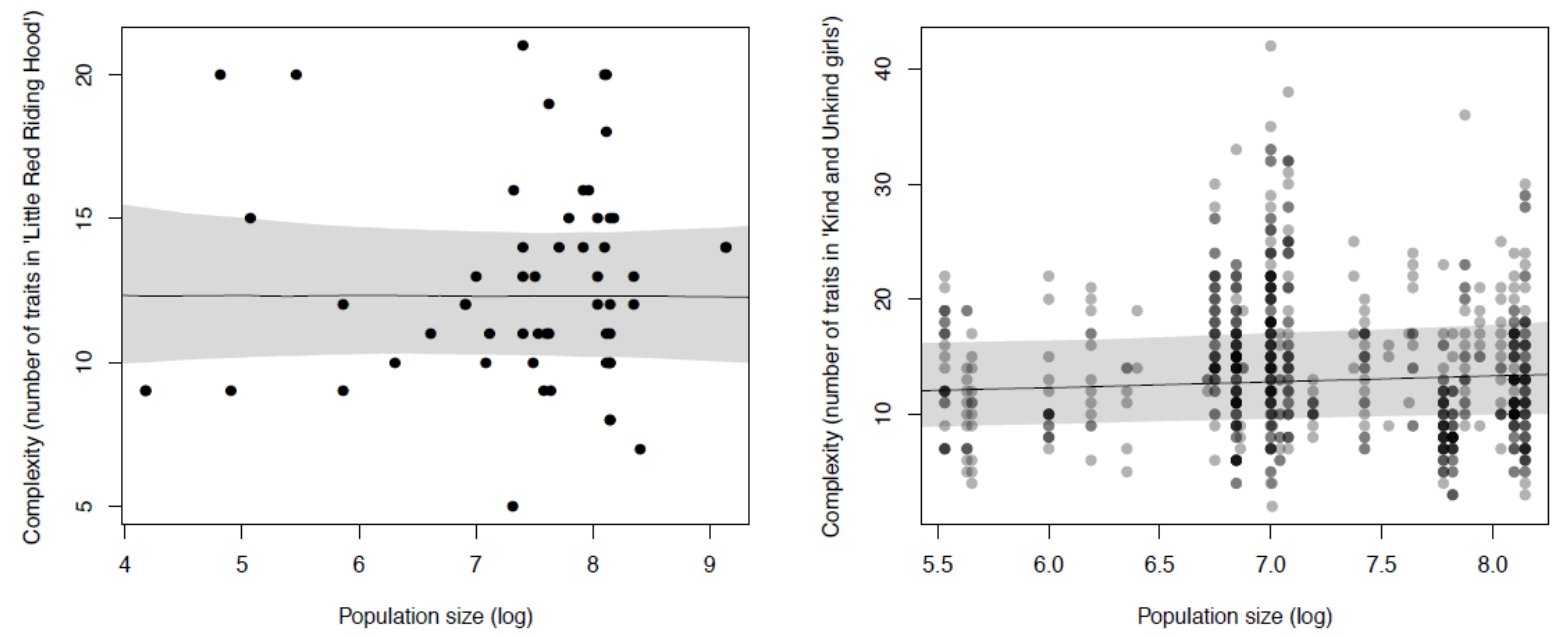

Figure 4 